\title{
A decade of perovskite photovoltaics
}

\author{
This year marks ten years of organic-inorganic perovskite solar cell research. Now, after achieving remarkable gains \\ in performance, applications are starting to make their way out of research laboratories into the real world.
}

n 2009, Tsutomu Miyasaka and colleagues in Japan reported on organic-inorganic lead halide perovskite compounds as light absorbers in dye-sensitized solar cells ${ }^{1}$. Although the properties of these materials had been investigated for many years and applications such as light-emitting diodes had been reported by Chondroudis and Mitzi in the United States ${ }^{2}$, the work of Miyasaka and team was the first to make use of perovskites for solar energy applications. However, as the device delivered just 3.8\% power conversion efficiency, had an active area of $0.24 \mathrm{~cm}^{2}$ and was stable for only a few minutes, these initial results presented uncertainties about the likely deployment of these materials in photovoltaics.

Nevertheless, given their interesting optoelectronic properties, research forged ahead and a few years later some seminal publications appeared. In 2012, the groups of Michael Grätzel in Switzerland and Nam-Gyu Park in South Korea ${ }^{3}$ demonstrated solidstate perovskite photovoltaic devices that overcame the poor stability of the material in liquid-based dye-sensitized solar cells. Later in the same year, Henry Snaith and colleagues in the United Kingdom demonstrated that the materials are not only able to sensitize a semiconductor, but also, and more interestingly, can themselves transport electronic charges to the solar cell electrodes, enabling higher device efficiencies ${ }^{4}$. Organicinorganic lead halide perovskites thus became the front runners among emerging photovoltaic materials and the field has advanced dramatically ever since.

Over the years, the perovskite community has put great efforts into addressing the peculiar physics and chemistry of these materials. For instance, the first Materials Research Society (MRS) symposium entirely dedicated to perovskite solar cells organized within the 2014 MRS fall meeting - discussed the causes of hysteresis in the current-voltage curves, the nature of charge traps, defects and grain boundaries, as well as ion migration, all of which posed threats to device stability and played a role in hampering device efficiency ${ }^{5}$. From there, striking progress has been made on the perovskite layer quality and device performances.

Quite remarkably, perovskite solar cells currently outperform the efficiency of more established photovoltaic technologies such as cadmium telluride and copper indium gallium selenide, although they lag behind silicon photovoltaics, which hold a $26.6 \%$ record efficiency. At the time of writing, the record perovskite power conversion efficiency, reported by the Chinese Academy of Sciences in late 2018 and certified by the National Renewable Energy Laboratory, is $23.7 \%$ (ref. ${ }^{6}$ ). Furthermore, small labscale cells have been scaled up to large-area devices $\left(>1 \mathrm{~cm}^{2}\right)$ and modules $\left(>10 \mathrm{~cm}^{2}\right)$, while employing deposition methods compatible with industrial manufacturing. Finally, perovskite solar cells have also now been demonstrated to be stable for thousands of hours under conditions that should mimic those that a real device can be exposed to.

Nevertheless, the lack of consistency in the stress factors used for assessing the stability of the devices has urged the perovskite community to identify degradation mechanisms and define test protocols that are specific for perovskites and would complement the specifications of standards defined by the International Electrotechnical Commission for photovoltaic technologies. Nature Energy is always on the lookout for these advances and seeks to support such endeavours wherever we can. Several such examples have been reported in our pages before. More widely, year by year, we have been gathering what we consider to be the most relevant research developments published across Nature Research journals in our 'Perovskites for optoelectronics' collection (https://www. nature.com/collections/fnnxcznnbb).

The remarkable developments on singlejunction perovskite solar cells over the past decade have also fuelled research on twojunction (or 'tandem') structures, where perovskite cells are coupled with other photovoltaic technologies. In 2018, Oxford PV, a UK-based company, announced a monolithic perovskite/silicon tandem solar cell with a certified $28.0 \%$ power conversion efficiency, outperforming both perovskite and silicon single-junction solar cells ${ }^{6}$. This should be compared with the current efficiency record for tandem solar cells of $32.8 \%$, held by LG Electronics for gallium indium phosphide/gallium arsenide monolithic devices, which are less cost effective than perovskites or silicon. This remarkable result sets great expectations for perovskite-based tandem cells to enter the market in the coming years. Indeed, a number of companies and research centres are devoted to technology transfer from laboratory to market, working on device stability and reliability, up-scaling and compatibility of the cell manufacturing with industrial processes, such as rollto-roll deposition.

Beyond multi-junction solar cells, building-integrated photovoltaics is another promising application. Saule Technologies has recently completed a pilot installation of semi-transparent perovskite photovoltaic devices into an office building façade in Poland ${ }^{7}$. Additionally, perovskite solar cells could be even more versatile: recent lab-based results show interesting performance under both concentrated and low-light intensities as well as good radiation tolerance, which are relevant to concentrator, indoor and space applications, respectively. But there is more to perovskites than photovoltaics: other perovskite-based applications, such as light-emitting diodes, detectors, lasers and photocatalysts, have been reported to hold great potential.

Yet for all these major advances, topical challenges still remain. These include device stability under realistic operational conditions, the environmental impact of the modules with concerns around lead and tin still being debated, the efficiency loss when upscaling to large-area devices, and remaining gaps in the fundamental materials physics. Encouragingly enough though, while silicon will keep dominating the photovoltaic market, perovskite solar cells seem likely to soon appear in commercial tandem solar cells and as building-integrated photovoltaic elements. We look forward to many more years of exciting discovery from these materials.

Published online: 14 January 2019

https://doi.org/10.1038/s41560-018-0323-9

\footnotetext{
References

1. Kojima, A. et al J. Am. Chem. Soc. 131, 6050-6051 (2009).

2. Chondroudis, K. et al. Chem. Mater. 11, 3028-3030 (1999).

3. Kim, H. S. et al. Sci. Rep. 2, 591 (2012).

4. Lee, M. M. et al. Science 338, 643-647 (2012).

5. Egger, D. A. et al. J. Phys. Chem. Lett. 6, 279-282 (2015).

6. Best Research-Cell Efficiencies (NREL, accessed 02 January 2019); https://www.nrel.gov/pv/assets/pdfs/pv-efficiencychart.20181221.pdf

7. Saule Technologies and Skanska change construction industry Saule Technologies https://sauletech.com/2018/12/13/saule-
} technologies-and-skanska-change-construction-industry (2018). 